\section{Two episodes of cutaneous non-tuberculous mycobacterial infection in a patient with psoriasis}

\author{
Wai Sze Agnes Chan, ${ }^{1}$ Shang-lan Tee, ${ }^{1}$ \\ Nisha Su Yien Chandran, ${ }^{2}$ Jiun Yit Pan ${ }^{1}$ \\ 'Department of Dermatology, National \\ Skin Centre; ${ }^{2}$ Department \\ of Dermatology, National University \\ Hospital, Singapore
}

\section{Abstract}

Non-tuberculous mycobacteria (NTM) are a group of environmental pathogens, which cause a broad spectrum of disease. The incidence of NTM infection is increasing, especially in immunocompromized patients. The past three decades also saw a rapid increase in the incidence of NTM infection involving otherwise healthy subjects. We report a case of cutaneous NTM infection in a 79-year-old Chinese woman, who was receiving methotrexate for psoriasis. Mycobacterial culture grew Mycobacterium abscessus, and the lesions cleared with a combination of oral clarithromycin, ciprofloxacin and doxycycline. Interestingly, she then developed a second episode of cutaneous NTM infection with Mycobacterium haemophilum over the same body region, five years after stoppage of methotrexate. Both episodes were separated in time and involved different species, indicating that they were independent from each other. We further discuss the risk factors for cutaneous NTM infection, treatment, and highlight the need for diagnostic vigilance.

\section{Case Report}

A 79-year-old Chinese female has been on follow-up at a specialist dermatological center in Singapore for psoriasis vulgaris. Following a pustular flare in October 2004, she was commenced on oral methotrexate (MTX) 2.5-7.5 mg weekly. A year later after having consumed a cumulative MTX dose of $390 \mathrm{mg}$, she noted the appearance of a new rash on the right antecubital fossa. She did not recall any preceding trauma at that region, including acupuncture or tattoos. Physical examination showed a $17 \times 10 \mathrm{~cm}$ erythematous coalescent ulcerated plaque studded with pustules. She was treated initially as for irritant contact dermatitis and erysipelas; however, there was no improvement despite six months of therapy with topical steroids and beta-lactam antibi- otics. A skin biopsy was then performed, which revealed a suppurative granulomatous infiltrate consisting of neutrophils, histiocytes and Langhans giant cells within the dermis (Figure 1). Acid-fast bacilli were not demonstrated on Ziehl-Neelsen stain; however tissue culture was positive for non-tuberculous mycobacteria (NTM), which was identified as Mycobacterium abscessus on polymerase chain reaction (PCR). MTX was stopped and she was prescribed a combination of oral clarithromycin $500 \mathrm{mg}$, ciprofloxacin $500 \mathrm{mg}$ and doxycycline $100 \mathrm{mg}$ twice daily for six months with complete resolution of the lesion.

There were no further infective episodes until August 2011, when she presented with another erythematous papulopustular rash over her right forearm and elbow (Figure 2). She had not received systemic immunosuppressive therapy in the past five years and she did not report any local trauma. There were no findings on history and physical examination to suggest an underlying immunodeficiency disorder. Laboratory tests including fasting blood glucose levels and HIV serology were normal, while total white cell count and differentials were within normal limits. Skin biopsy revealed a dermal granulomatous dermatitis (Figure 3). Tissue culture confirmed the presence of NTM, identified as Mycobacterium haemophilum on PCR. She was treated with clarithromycin $500 \mathrm{mg}$ and ciprofloxacin $500 \mathrm{mg}$ twice daily with complete clearance of the lesion after ten months. She remains well on her last follow-up visit with us.

\section{Discussion}

Non-tuberculous mycobacteria are free living, fastidious aerobic acid fast bacilli organisms widely distributed in the environment. Six clinical syndromes account for most infections caused by NTM including pulmonary disease, lymphadenitis, skin or soft tissue infections, skeletal (bone, joint, tendon), foreign body and central venous catheter infection, and disseminated diseases. ${ }^{1}$ Infection is more common in patients who are immunocompromized. Runyon identified and divided NTM into slow growing groups, basing on their ability to produce pigment, and rapidly growing groups (RGM) such as M. abcessus, M. fortuitum, and M. chelonae. ${ }^{2}$ The most common NTM species to cause cutaneous disease are $M$. marinum and RGM. ${ }^{3}$

Recent epidemiological studies have confirmed that the incidence of cutaneous NTM infection is increasing worldwide, highlighting the importance of recognizing this entity. ${ }^{4,3}$ The rising incidence has been attributed to improved diagnostic techniques as well as the popularity of cosmetic and medical procedures,
Correspondence: Wai Sze Agnes Chan, Department of Dermatology, National Skin Centre, 1 Mandalay Road, 308205 Singapore.

Tel.: +65.9364.7239 - Fax: +65.6352.3225.

E-mail: agneschan@nsc.gov.sg

Key words: Mycobacterium abscessus Mycobacterium hemophilum; cutaneous; non tuberculous mycobacteria; psoriasis.

Contributions: the authors contributed equally.

Conflict of interest: the authors declare no potential conflict of interest.

Received for publication: 10 November 2014.

Revision received: 30 March 2015.

Accepted for publication: 26 April 2015.

This work is licensed under a Creative Commons Attribution NonCommercial 3.0 License (CC BYNC 3.0).

(C) Copyright W.S.A. Chan et al., 2015

Licensee PAGEPress, Italy

Dermatology Reports 2015; 7:5712

doi:10.4081/dr.2015.5712

which carry a risk of contamination. Cutaneous infection presents with myriad clinical signs ranging from erythematous plaques, papules or pustules to subcutaneous nodules, abscesses and ulcers. Direct inoculation via penetrating injury is an important route of infection, ${ }^{4}$ and most cases involve the extremities. Unfortunately a history of antecedent trauma is not always elicited or may be missed, especially given the long incubation period of certain organisms (up to 12 months for M. abscessus). ${ }^{5}$ This was the case of our patient, whose disease was picked up on skin biopsy only after six months of empirical treatment. AFB staining has a low yield of $36.2 \%,{ }^{2}$ and identification of NTM species may require PCR testing. Indeed, misdiagnosis occurs in up to $82 \%$ of cases,${ }^{6}$ emphasizing the need for a high index of suspicion, requesting for mycobacterial culture, and repeating biopsies in clinical scenarios where a patient may be at risk.

Immunosuppression is an important risk factor for NTM infection and is likely to have contributed to our patient's initial episode, having occurred after 14 months of therapy with MTX. Although her cumulative dose was only $390 \mathrm{mg}$, it has been noted that even low doses of MTX may induce B cell suppression and inhibit differentiation, leading to susceptibility to infection. ${ }^{7}$ Opportunistic infections such as Pneumocystis carinii pneumonia, Mycobacterium avium-intracellulare pneumonia and disseminated histoplasmosis have been reported after MTX therapy as short as 11 weeks to 17 years duration. ${ }^{8}$ In immunocompromized hosts, cutaneous NTM infection 
presents more frequently, sometimes without associated inciting skin injury, and often involving multiple sites and/or deeper tissues. Our patient was infected with a second episode of cutaneous NTM with a different species with $M$. Haemophilum; hence the two episodes are distinct and not due to re-activation of latent infection. An inciting factor for the second episode is less obvious given the absence of trauma or drug-induced immunosuppression. Moreover, patients with psoriasis usually do not carry an increased risk of skin infection, due to up-regulation of antimicrobial peptides and cathelicidins, compared to patients with eczema or those with normal skin. ${ }^{9}$ An underlying immunodeficiency syndrome, previously reported in other families susceptible to mycobacteria, ${ }^{10,11}$ seems unlikely in our patient given the absence of other recurrent infections to suggest defects in her cellular immune response. We instead postulate that her advanced age, along with the presence of dry and fissured psoriatic plaques, has led to skin barrier problems causing an increase in skin fragility, thereby compromising the innate immunity of skin and facilitating entry of mycobacteria with consequent infection.

The importance of diagnostic vigilance is crucial, as a delay of up to 7.1 months from initial clinical presentation to commencement of treatment has been reported. ${ }^{6}$ The choice of antimicrobial treatment depends on species identification and the outcome of susceptibility testing. Most studies recommend clarithromycin alone or in combination with fluoroquinolones or tetracyclines for 4 to 6 months to treat rapid-growing mycobacteria such as $M$. abscessus, with a curative rate of $87 \% .^{12} \mathrm{M}$. Haemophilum is resistant to isoniazid and ethambutol in vitro, and experts recommend a combination of clarithromycin, ciprofloxacin and a rifamycin antibiotic. Optimal duration of therapy is not known, and common recommendation is to continue treatment for 4 to 6 weeks upon resolution of cutaneous lesion.,13 Immunocompromized patients have cutaneous lesions which lasts several months longer than immunocompetent patients and are at higher risk of complications such as deep tissue infections, ${ }^{14}$ duration of treatment should therefore be longer with evaluation for surgical intervention including incision and drainage.

\section{Conclusions}

Non tuberculous mycobacterium organisms are widespread in the environment, and exposure is common. We have encountered a patient with psoriasis who was treated for two separate episodes of cutaneous NTM infection; first while she was receiving immunosuppres-

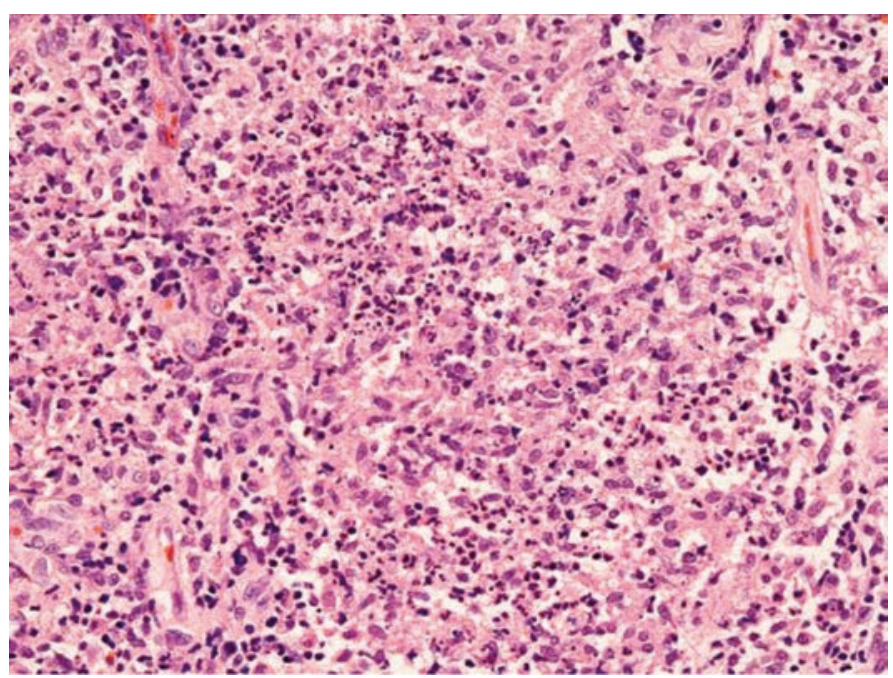

Figure 1. This is composed of suppurative granulomas containing aggregates of neutrophils surrounded by histiocytes and lymphocytes (Hematoxylin and Eosin, 200x).

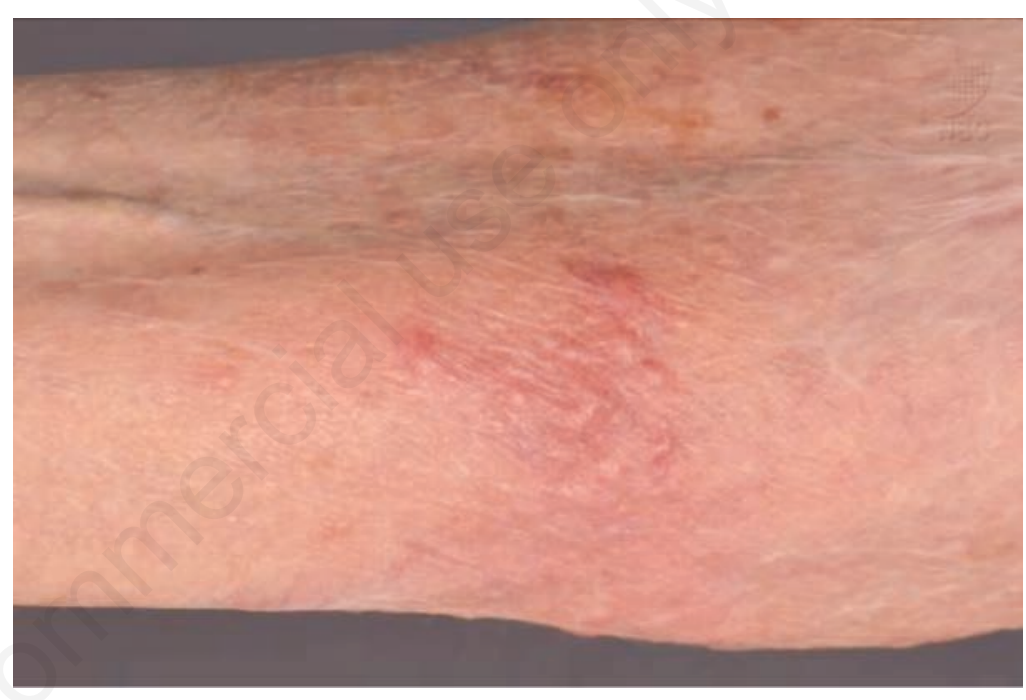

Figure 2. Erythematous papulopustular plaque at flexor surface of the right forearm.

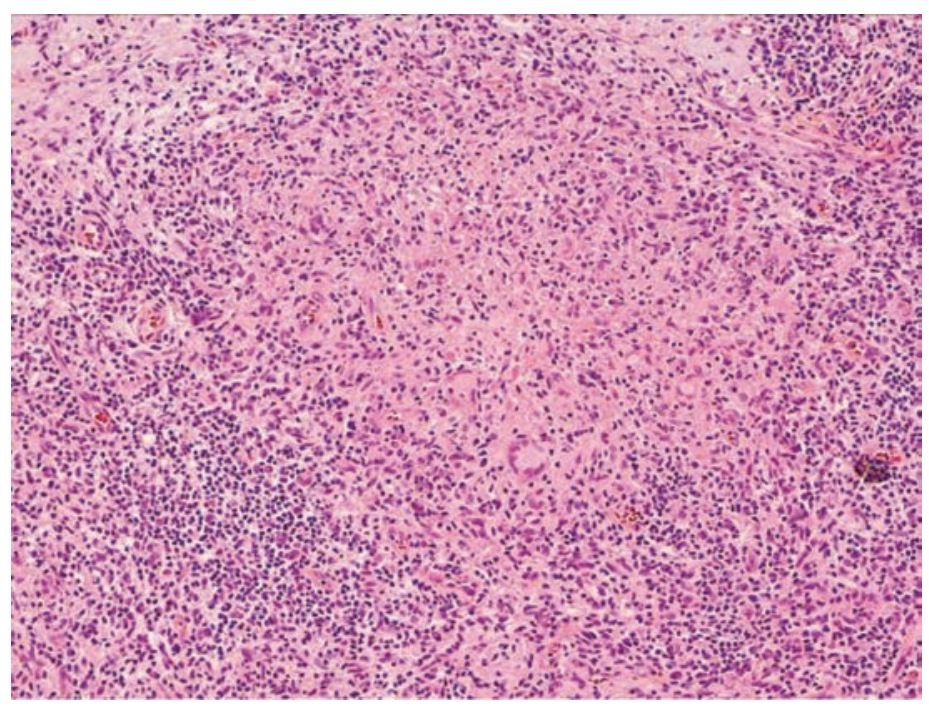

Figure 3. Higher power reveals an infiltrate of histiocytes admixed with Langhans-type giant cells and surrounded by numerous lymphocytes, plasma cells and neutrophils (Hematoxylin and Eosin, 100x). 
sive therapy, and second, much later on, when she was apparently healthy with no known predisposing factors, with $M$. abscessus and $M$. Haemophilum respectively.

We propose that she was susceptible to cutaneous NTM infection due to advanced age and defective skin barrier, leading to implantation of NTM organism to the skin. Immunosuppressive therapy was an additional risk factor in the first episode. With the rise in incidence of cutaneous NTM infection, physicians must have a high index of suspicion, especially in patients with non-resolving lesions of the upper distal extremities with a history of antecedent trauma, including acupuncture or medical procedures.

\section{References}

1. No authors listed. Diagnosis and treatment of disease caused by nontuberculous mycobacteria: this official statement of the American Thoracic Society was approved by the Board of Directors, March 1997. Medical Section of the American Lung Association. Am J Respir Crit Care Med
1997;156:S1-25.

2. Runyon EH. Anonymous mycobacteria in pulmonary disease. Med Clin North Am 1959;43:273-304.

3. Hsiao CH, Tsai TF, Hsueh PR. Characteristics of skin and soft tissue infection caused by non-tuberculous mycobacteria in Taiwan. Int J Tuberc Lung Dis 2011;15:811-7.

4. Wentworth AB, Drage LA, Wengnack NL, et al. Increased incidence of cutaneous nontubeculous mycobacterial infection, 1980 to 2009: a population- based study. Mayo Clin Proc 2013;88:38-45.

5. Fitzgerald DA, Smith AG, Lees A, et al. Cutaneous infection with mycobacterium abscessus. Br J Dermatol 1995;132:800-4.

6. Dodiuk-Gad R, Dyachenko P, Ziv M, et al. Nontuberculous mycobacterial infections of the skin: a retrospective study of 25 cases. J Am Acad Dermatol 2007;57:413-20.

7. Olsen NJ, Callahan LF, Pincus T. Immunologic studies of rheumatoid arthritis patients treated with methotrexate. Arthritis Rheum 1987;30:481-8.

8. LeMense GP, Sahn SA. Opportunistic infection during treatment with low dose methotrexate. Am J Respir Crit Care Med
1994;150:258-60

9. Morizane S, Gallo RL. Antimicrobial peptides in the pathogenesis of psoriasis. $\mathrm{J}$ Dermatol 2012;39:225-30.

10. Newport MJ, Huxley CM, Huston S, et al. A mutation in the interferon gamma receptor gene and susceptibility to mycobacterial infection. N Engl J Med 1996;335:1941-9.

11. Chetchotisakd P, Mootsikapun P, Anunnatisiri S, et al. Disseminated infection due to rapidly growing mycobacteria in immunocompetent hosts presenting with chronic lymphadenopathy: a previous unrecognized clinical entity. Clin Infect Dis 2000;30:29-34.

12. Kothavade RJ, Dhurat RS, Mishra SN, et al. Clinical and laboratory aspects of the diagnosis and management of cutaneous and subcutaneous infections caused by rapidly growing mycobacteria. Eur J Clin Microbiol Infect Dis 2013;32:161-88.

13. Wagner D, Young LS. Nontuberculous mycobacterial infections: a clinical review. Infection 2004;32:257-70.

14. Lee WJ, Kang SM, Sung H, et al. Nontuberculous mycobacterial infections of the skin: a retrospective study of 29 cases. J Dermatol 2010;37:965-72. 